\title{
Interpreting Umbilical Cord Blood Gases: Section 7: Fetal Circulatory Failure, Part II
}

Jeffrey Pomerance, MD, MPH

"On the day of admission, the mother presented at the hospital because of spontaneous rupture of membranes with the escape of clear fluid confirmed by examination. Although the mother reported normal fetal movement, the FHR tracing revealed a baseline of 160 bpm with minimal variability and absent accelerations."

Case 20: Chronic Fetal Heart Failure: Unknown Etiology

The mother was a 34-year-old, A+, antibody negative, gravida 2, para 1, aborta 0 , with an intrauterine pregnancy at $396 / 7$ weeks gestation. Approximately two weeks before admission, the mother reported decreased fetal movement. An NST was reactive, demonstrating a baseline FHR of $135 \mathrm{bpm}$ with accelerations to 155-165 bpm. On the day of admission, the mother presented at the hospital because of spontaneous rupture of membranes with the escape of clear fluid confirmed by examination. Although the mother reported normal fetal movement, the FHR tracing revealed a baseline of $160 \mathrm{bpm}$ with minimal variability and absent accelerations. Excessive uterine activity was present with five to six contractions in eight minutes. Intermittently, the tracing revealed a pattern of smooth oscillations (sinusoidal pattern). At approximately 15 minutes prior to delivery, the FHR fell slowly to 50-60 $\mathrm{bpm}$, and an emergent cesarean section was called with prompt delivery under general anesthesia. Upon entering the uterine cavity, the amniotic fluid was dark and blood-stained. Upon delivery of the infant, the placenta was found free-floating in the uterine cavity. The 3970-gram infant's Apgar scores were 0, 0, 0, 1, 3, and 5 at one, five, 10,15, 19, and 35 minutes, respectively.

Cord blood gas results were as follows:

\begin{tabular}{|c|c|c|}
\hline & Umbilical Vein & Umbilical Artery \\
\hline $\mathrm{pH}$ & 7.24 & 6.91 \\
\hline $\mathrm{Pco}_{2} \underset{(\mathrm{kPa})}{(\mathrm{mmHg})}$ & $\begin{array}{l}54 \\
7.20\end{array}$ & $\begin{array}{l}105 \\
14.00\end{array}$ \\
\hline $\mathrm{Po}_{2} \underset{(\mathrm{kPa})}{(\mathrm{mmHg})}$ & $\begin{array}{l}39 \\
5.20\end{array}$ & $\begin{array}{l}9 \\
1.20\end{array}$ \\
\hline $\mathrm{BD}^{*}$ (mmol/L) & 4 & 12 \\
\hline
\end{tabular}

The hematocrit was 52\% (approximately the same at follow-up). Extensive resuscitation included suctioning, intubation, ventilation with $100 \%$ oxygen, chest compressions, five doses of epinephrine via ETT, two doses of sodium bicarbonate, and $40 \mathrm{~mL}$ of normal saline through a peripheral IV. At age 35 minutes, the still flaccid infant began gasping.

\section{"Extensive resuscitation included suctioning, intubation, ventilation with $100 \%$ oxygen, chest compressions, five doses of epinephrine via ETT, two doses of sodium bicarbonate, and $40 \mathrm{~mL}$ of normal saline through a peripheral IV. At age 35 minutes, the still flaccid infant began gasping."}

An $A B G$ at age 48 minutes revealed the following results:

\begin{tabular}{|c|c|}
\hline & Infant's ABG \\
\hline pH & 6.86 \\
\hline $\mathrm{PCO}_{2} \underset{(\mathrm{kPa})}{(\mathrm{mmHg})}$ & $\begin{array}{l}102 \\
13.60\end{array}$ \\
\hline $\begin{array}{ll}\mathrm{Po}_{2} & (\mathrm{mmHg}) \\
(\mathrm{kPa}) & \end{array}$ & $\begin{array}{l}105 \\
14.00\end{array}$ \\
\hline BD (mmol/L) & 15 \\
\hline
\end{tabular}

The placenta weighed $760 \mathrm{~g}$ and showed mild acute chorioamnionitis and a small area of old infarct $(1.1 \mathrm{~cm}$ in greatest diameter). No blood clot or other indicator of placental abruption was deduced from inspection of the maternal surface of the placenta.

The infant continued to be flaccid and had no spontaneous movements; pupils were fixed at $2 \mathrm{~mm}$. ETT suctioning resulted in dark, old blood. Because of poor ventilation, lung lavage was performed twice and resulted in improved chest movement. The abdomen appeared "round and hydropic."

Seizures began prior to three hours of age and were treated with phenobarbital. The newborn's base deficit normalized after eight hours of age. The initial corrected WBC count at two to three hours of age was $19,400 / \mathrm{mm}^{3}$ with $4 \%$ bands, $44 \%$ neutrophils, and $165 \mathrm{NRBCs} / 100$ WBCs). NRBCs were approximately $32,000 /$ $\mathrm{mm}^{3}(19,400 / 100 \times 165)$. The infant developed evidence of DIC and hepatic and renal dysfunction. An EEG showed a burst suppression pattern. A CT of the head at approximately 19 hours of age was normal. An MRI and MRA of the head at about age 24 hours demonstrated a question of diffusion abnormalities bilaterally in the ventral lateral thalami and the subcortical white matter. At age four days, an MRI of the brain demonstrated restricted diffusion in the lateral basal ganglia regions. At age four years, this child carried diagnoses of dystonia, spastic quadriparesis, and developmental delay.

\section{Interpretation}

The umbilical cord venous blood sample demonstrates a mild respiratory acidosis and a borderline high $\mathrm{PO}_{2}$, a finding associated with cord occlusion, chronic fetal heart failure (see Case 19), and a normal base deficit. The venous $\mathrm{pH}$ is borderline low. The umbil- 
ical cord arterial blood gas sample demonstrates severe respiratory and mild to moderate metabolic acidosis. The umbilical venoarterial blood gas differences are quite widened, suggesting either cord compression (common) or fetal heart failure (rare). However, the findings during labor suggest placental abruption - excessive spontaneous uterine activity, bloody amniotic fluid upon entry into the uterus (it was clear initially), and the free-floating placenta encountered immediately after delivery of the infant. In the face of uteroplacental insufficiency induced by placental abruption, one would expect to see approximately equal derangements of both umbilical venous and arterial blood gas samples (see Case 8), clearly dissimilar from the values that are present in this case. Initially, I thought these cord gas results must have belonged to a different newborn, an occurrence that is always possible but very unlikely, and the last bastion for unexplainable cord blood gas results.

The diagnosis of abruption is a clinical one, and the absence of fresh clots behind the placenta at the time of delivery does not exclude acute abruption. (1) With chronic placental abruption, one would expect confirmation from pathological examination of the placenta. Further, the amniotic fluid was clear when the membranes ruptured. Therefore, it seems likely that acute placental abruption began sometime after the onset of labor (or was the initiator of labor) and became complete around the time of delivery.

\section{"On admission, a maternal report of "normal" fetal movement seems improbable given the FHR pattern of decreased variability and absent accelerations. Often mothers feel "chided" when their perception of decreased fetal movement appears to be in error. This may result in a future reluctance to report any decrease in fetal movement."}

On admission, a maternal report of "normal" fetal movement seems improbable given the FHR pattern of decreased variability and absent accelerations. Often mothers feel "chided" when their perception of decreased fetal movement appears to be in error. This may result in a future reluctance to report any decrease in fetal movement. When the mother was reassured after testing, no explanation was offered as to the cause of her perceived change in fetal behavior. No ultrasound examination was performed to evaluate the amount of amniotic fluid.

Placental pathology showed mild acute chorioamnionitis; however, chorioamnionitis with or without funisitis does not appear to influence cord $\mathrm{pH}$ or base deficit.

The upper limit of normal NRBC shortly after birth is approximately $1000 / \mathrm{mm}^{3}$. (2) The extremely elevated NRBC count of $32,000 / \mathrm{mm}^{3}$ suggests fetal hypoxemia over at least days rather than hours. Other potential explanations exist for the very large differences between the umbilical venous and arterial samples. However, all of the other potential explanations involve cord occlusion of one sort or another. Chronic cord occlusion with a slowly tightening knot in the umbilical cord provides a possible explanation; however, no knot was found, and variable decelerations were absent. Similarly, the explanation of occult cord prolapse seems improbable due to the absence of both variable decelerations or a finding of any unusual cord location at the time of the emergency cesarean delivery. An occult cord is frequently missed at the time of emergent cesarean section. The major reasons arguing against these mechanisms of fetal embarrassment include the smoking gun in this case, the hydropic appearing neonatal abdomen, a classic sign of fetal heart failure. (3) It is hard to ignore this finding. Regardless of the cause, fetal heart failure was present prior to the onset of labor. Furthermore, there was an intermittent FHR sinusoidal pattern during labor. This instance is the third fetus I am aware of in whom a sinusoidal FHR pattern was observed in the absence of fetal anemia and the presence of fetal heart failure. Perhaps it is fetal heart failure rather than severe fetal anemia that is the prime cause of a fetal sinusoidal heart rate pattern.

In summary, although there was clinical evidence of placental abruption, this development likely did not play a major role during labor and delivery. Instead, chronic fetal heart failure of undetermined etiology was responsible for the terminal events and the extremely disparate umbilical cord blood gas results. The mechanism by which umbilical cord blood gases become widely disparate when the fetus has heart failure is explained in the previous case.

Even though the apparent cause of the initial Apgar score of zero was chronic fetal heart failure, if this had not been present, a complete placental abruption would likely have been catastrophic all by itself.

\section{Key Points}

- $\quad$ Combined venous and arterial umbilical cord blood gases often provide a different and improved view of the pathophysiology of fetal compromise.

- $\quad$ A fetal sinusoidal heart rate pattern may be primarily associated with fetal heart failure rather than only severe fetal anemia.

\section{References:}

1. Benirschke K, Kaufman P. Hypertensive disorders. In: Pathology of the Human Placenta, $2^{\text {nd }}$ edition. New York: Springer-Verlag, 1990, pp511-2.

2. Hermansen MC. Nucleated red blood cells in the fetus and newborn. Arch Dis Child Neonatal Ed. 2001;84:F211-5.

3. Ojala TH, Hornberger LK. Fetal heart failure. Front Biosci (Schol Ed) 2010;2:891-906.

NT

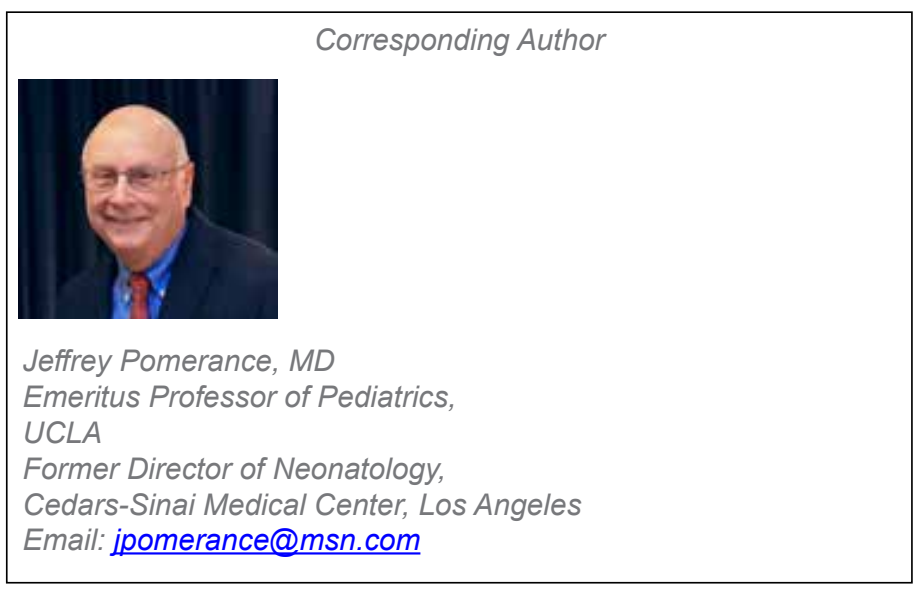

